\title{
A study on the prevention and treatment of murine calvarial inflammatory osteolysis induced by ultra-high-molecular-weight polyethylene particles with neomangiferin
}

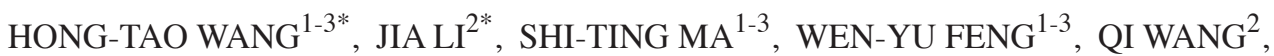 \\ HONG-YAN ZHOU ${ }^{2}$, JIN-MIN ZHAO ${ }^{1-3}$ and JUN YAO ${ }^{1-3}$ \\ ${ }^{1}$ Research Centre for Regenerative Medicine and Guangxi Key Laboratory of Regenerative Medicine; \\ ${ }^{2}$ Orthopedic Department, The First Affiliated Hospital of Guangxi Medical University; ${ }^{3}$ Collaborative Innovation Center of \\ Guangxi Biological Medicine, Guangxi Medical University, Nanning, Guangxi 530021, P.R. China
}

Received February 28, 2018; Accepted August 9, 2018

DOI: $10.3892 /$ etm.2018.6725

\begin{abstract}
The present study aimed to examine the influence of neomangiferin on murine calvarial inflammatory osteolysis induced by ultra-high-molecular-weight polyethylene (UHMWPE) particles. Eight-week-old male C57BL/J6 mice served as an inflammatory osteolysis model, in which UHMWPE particles were implanted into the calvarial subperiosteal space. The mice were randomly distributed into four groups and treated with different interventions; namely, a sham group [phosphate-buffered saline (PBS) injection and no UHMWPE particles], model group (PBS injection and implantation of UHMWPE particles), low-dose neomangiferin group (UHMWPE particles $+2.5 \mathrm{mg} / \mathrm{kg}$ neomangiferin), and high-dose neomangiferin group (UHMWPE particles $+5 \mathrm{mg} / \mathrm{kg}$ neomangiferin). Following 3 weeks of feeding according to the above regimens, celiac artery blood samples were collected for
\end{abstract}

Correspondence to: Dr Jun Yao or Dr Jin-Min Zhao, Research Centre for Regenerative Medicine and Guangxi Key Laboratory of Regenerative Medicine, Guangxi Medical University, 22 Shuangyong Road, Nanning, Guangxi 530021, P.R. China

E-mail: yaojun800524@163.com

E-mail: zhaojinmin@126.com

*Contributed equally

Abbreviations: AL, aseptic loosening; $\mathrm{BMD}$, bone mineral density; BV/TV, bone volume/tissue volume ratio; ELISA, enzyme-linked immunosorbent assay; H\&E, hematoxylin and eosin; micro-CT, micro-computed tomography; PBS, phosphate-buffered saline; TRAP, tartrate-resistant acid phosphatase; UHMWPE, ultra-high-molecular-weight polyethylene; RANKL, receptor activator of nuclear factor- $\mathrm{\kappa B}$ ligand; OSCAR, osteoclast-related receptor; CTX-1, cross-linked C-telopeptide of type I collagen; OPG, osteoprotegerin

Key words: neomangiferin, osteoclast, inflammatory osteolysis, ultra-high-molecular-weight polyethylene particle an enzyme-linked immunosorbent assay (ELISA) to determine the expression of receptor activator of nuclear factor- $\kappa \mathrm{B}$ ligand (RANKL), osteoclast-related receptor (OSCAR), cross-linked C-telopeptide of type I collagen (CTX-1); osteoprotegerin (OPG), tumor necrosis factor (TNF)- $\alpha$, and interleukin (IL)-1 $\beta$. Subsequently, the mice were sacrificed by cervical dislocation following ether-inhalation anesthesia, and the skull was separated for osteolysis analysis by micro-computed tomography (micro-CT). Following hematoxylin and eosin staining, tartrate-resistant acid phosphatase (TRAP) staining was performed to observe the dissolution and destruction of the skull. The micro-CT results suggested that neomangiferin significantly inhibited the murine calvarial osteolysis and bone resorption induced by UHMWPE particles. In addition, the ELISA results showed that neomangiferin decreased the expression levels of osteoclast markers RANKL, OSCAR, CTX-1, TNF- $\alpha$ and IL-1 $\beta$. By contrast, the levels of OPG increased with the neomangiferin dose. Histopathological examination revealed that the TRAP-positive cell count was significantly reduced in the neomangiferin-treated animals compared with that in the positive control group, and the degree of bone resorption was also markedly reduced. Neomangiferin was found to have significant anti-inflammatory effects and to inhibit osteoclastogenesis. Therefore, it has the potential to prevent the aseptic loosening of a prosthesis following artificial joint replacement.

\section{Introduction}

Currently, it is suggested that the main reason for aseptic loosening (AL) of an artificial joint prosthesis is due to an inflammatory osteolytic reaction induced by wear particles $(1,2)$. Studies have suggested that wear particles, including metal particles (titanium, cobalt), ultra-high-molecular-weight polyethylene (UHMWPE), and polymethylmethacrylate, are largely responsible for the initial stage and progression of an inflammatory osteolytic reaction (3-6). A chronic inflammatory reaction induced by wear particles triggers macrophage infiltration and cytokine release associated with osteolysis, suppression of osteoblast proliferation and differentiation, and promotion 
of osteoclast activation. Therefore, osteoclast differentiation induced by wear particles is a determinant of inflammatory osteolysis (7-10). The receptor activator of nuclear factor- $\kappa \mathrm{B}$ (RANK)-RANK ligand (RANKL)-osteoprotegerin (OPG) signal transduction pathway is a crucial signaling pathway for osteoclast differentiation and maturation and influences the key process of osteolysis around the prosthesis. Medication inhibiting osteoclast activity is an effective method of treating osteolysis. Drugs, including estrogen and bisphosphonates, can inhibit bone resorption; however, significant side effects limit their long-term use. There is a herbal decoction that inhibits osteoclast action in the treatment of osteoporosis, in which neomangiferin is one of the important active ingredients. Neomangiferin, derived from Anemarrhena plants, has a molecular formula of $\mathrm{C}_{25} \mathrm{H}_{28} \mathrm{O}_{16}$ and molecular weight of 584.4802. In addition, neomangiferin has anti-inflammatory, antioxidant, anti-osteoporotic, and liver and kidney protective biological activities (11-14). In our previous experiments (Wang et al; unpublished data), it was found that neomangiferin inhibited osteoclast differentiation in vitro, however, the exact mechanism remains to be elucidated. Therefore, the purpose of the present study was to investigate the role of neomangiferin in the inhibition of inflammatory osteolysis through in vivo animal experiments and to examine the possible mechanism of action of neomangiferin, thereby providing therapeutic options for the prevention or treatment of metabolic bone diseases induced by wear particles.

\section{Materials and methods}

Preparation of the UHMWPE particle suspension. Pure UHMWPE particles were purchased from Germany Clariant (Gersthofer, Germany). The average particle diameter was $1.84 \pm 1.50 \mu \mathrm{m}$. It was estimated that $>32 \%$ of the particles were $<1 \mu \mathrm{m}$ in size. The UHMWPE particles were soaked in $75 \%$ ethanol for $48 \mathrm{~h}$ to remove toxins; subsequently, the cells were cryogenically sealed with standard ethylene oxide. The UHMWPE particles were cleaned three times with phosphate-buffered saline (PBS) and formulated into a 100-mg/ml UHMWPE particle suspension with cryogenic PBS prior to use.

Animals. The animal model was designed according to previous reports $(15,16)$. The neomangiferin was screened for osteoclast differentiation, and it was found that the neomangiferin inhibited the formation of osteoclasts at a concentration of $2.5 \mu \mathrm{mol} / 1$ (data not shown). The concentration of Chinese herbs required to inhibit osteoclasts in vivo is a low concentration of $1-10 \mathrm{mg} / \mathrm{kg}$ and a high concentration of 2-30 mg/kg (17-18). In the present study, the doses in vivo were calculated (low and high concentrations of 2.5 and $5 \mathrm{mg} / \mathrm{kg}$, respectively) according to the weight of mice and the content of body fluid (data not shown). A total of 24, 8 week old C57BL/6 mice (specific pathogen free grade) were provided by the Experimental Animal Center of Guangxi Medical University (Nanning, China). Mice were housed at a temperature of $22-24^{\circ} \mathrm{C}$, a humidity of $56 \%$ and interval lighting (12 h dark/light cycle), with regular ventilation. All mice were fed standard laboratory chow with ad libitum water, but were fasted from 10:00 to 15:00 prior to experimentation. The animals were randomly divided into four groups ( $n=6$ per group): Negative control group (injected with PBS only); positive control group (UHMWPE particles + PBS); neomangiferin (purity $>98 \%$ by high-performance liquid chromatography; Chengdu Manster Biotechnology Co., Ltd., Sichuan, China), low-dose group (UHMWPE particles $+2.5 \mathrm{mg} / \mathrm{kg}$ neomangiferin), and neomangiferin high-dose group (UHMWPE particles $+5 \mathrm{mg} / \mathrm{kg}$ neomangiferin). The animal experimental protocol was approved by the Animal Ethics Committee of Guangxi Medical University (approval no. 201707006), and the Guidelines for Care and Use of Laboratory Animals were strictly followed.

According to the body weight of each mouse, anesthesia was induced intraperitoneally with $4 \%$ chloral hydrate (400 mg/kg mouse body weight). Following successful anesthesia, a depilatory agent was used to adequately remove iodine from the hair of the mouse. Following placement on a disposable sterile towel, the mouse skull sagittal line and mouse bilateral external auditory canal connection was selected as a reference point. Subsequently, via the midpoint of the connection, incisions of the skin and subcutaneous tissue were made using 15 small circular knives. Mouse calvarial bone was fully exposed in an area of $\sim 1 \times 1 \mathrm{~cm}$ of full-range periosteum. A $2 \times 2 \mathrm{~mm}$ periosteum defect area was created using a small round knife at the top of the cranium. The control group was syringe-injected with sterile PBS (100 $\mu 1)$; the other three groups were injected with UHMWPE particles (100 $\mu 1$, $100 \mathrm{mg} / \mathrm{ml}$ ). The skin wound was closed with sutures postoperatively to prevent drug spillover. Following these procedures, the mice were housed separately. Penicillin was routinely used to ensure anti-infective surgery. At 2 days post-surgery, the negative control group and the positive control group were injected with $100 \mu \mathrm{l}$ PBS; the other two groups were injected with neomangiferin at 2.5 or $5 \mathrm{mg} / \mathrm{kg}$. The drug was injected every other day for 21 days. None of the mice died; therefore, the model was successful $(19,20)$. At 3 weeks post-surgery, each group of mice was anesthetized with ether, and blood samples from the celiac artery were collected for ELISA assays. The mice were sacrificed by cervical dislocation, following which the intact skull was collected, trimmed, and immersed in $10 \%$ paraformaldehyde for fixing and later use.

Serum ELISA. In each group, blood was collected from the celiac artery of the mouse. Subsequently, serum was prepared via centrifugation $\left(350 \mathrm{x}\right.$ g for $10 \mathrm{~min}$ at $4^{\circ} \mathrm{C}$ ) and analyzed using an ELISA kit (Wuhan Landing Medical Hi-Tech Co., Ltd., Hubei, China). The levels of osteoclast-related receptor (OSCAR), RANKL, cross-linked C-telopeptide of type I collagen (CTX-1), OPG, interleukin $1 \beta$ (IL-1 $\beta$ ), and tumor necrosis factor (TNF)- $\alpha$ were assayed.

Micro-computed tomography (micro-CT) examination. Following fixing in paraformaldehyde $(40 \mathrm{~g} / \mathrm{l})$ solution for 1 day, the skulls from each group, with UHMWPE particles removed, were scanned by micro-CT (Skyscan1176; Bruker microCT, Kontich, Belgium). The parameters were set as follows: Resolution $18 \mu \mathrm{m}$, current $100 \mathrm{~mA}$, voltage $80 \mathrm{kV}$, and exposure time $100 \mathrm{~ms}$. Bone mineral density (BMD) and the bone volume/tissue volume ratio (BV/TV) were analyzed by software measurement (Skyscan1176; Skyscan CT analyser v1.115.2.2+; Bruker microCT). Bone resorption pits and porosity were quantified using Image $\mathrm{J}$ software (version 1.36; NIH, Bethesda, MA, USA). 

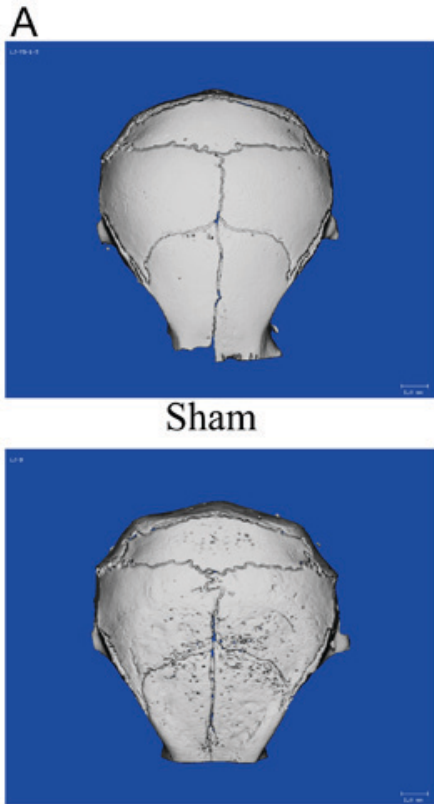

L-dose

B
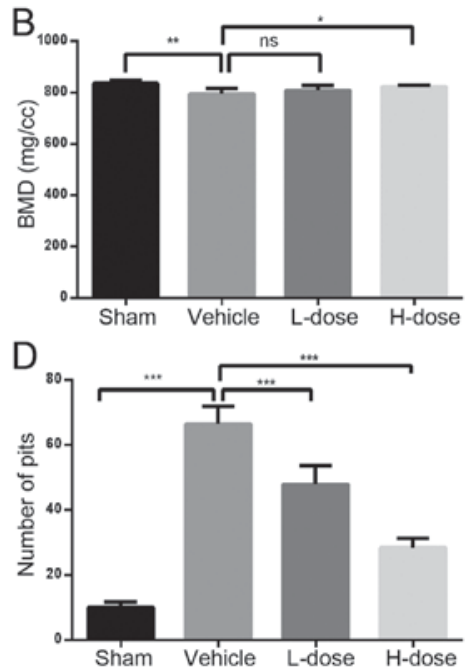

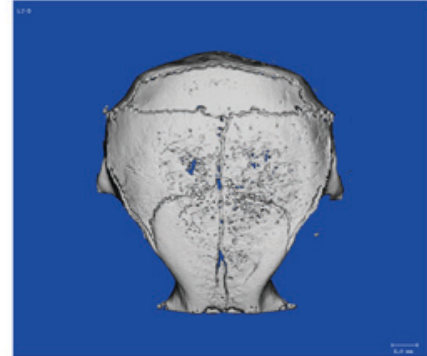

Vehicle

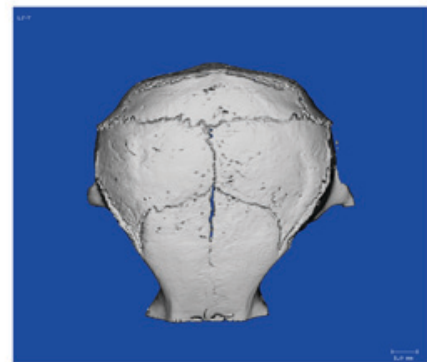

H-dose
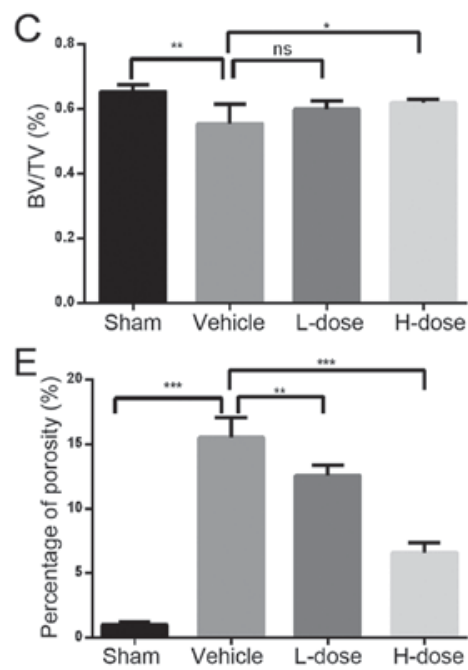

Figure 1. Neomangiferin inhibits UHMWPE particle-induced calvarial osteolysis in mice. (A) Micro-computed tomography scans of the chondral callus induced by UHMWPE particles in each group. (B) BMD, (C) BV/TV, (D) pit number, (E) porosity percentage of each experimental specimen (within ROI, 6x6 mm); n=6. Following one-way analysis of variance, an SNK-q test was performed to determine statistical significance. Results are expressed as the mean \pm standard deviation $\left({ }^{*} \mathrm{P}<0.05,{ }^{* *} \mathrm{P}<0.01\right.$ and ${ }^{* * * *} \mathrm{P}<0.001$, compared with the positive control group). UHMWPE, ultra-high-molecular-weight polyethylene; ns, no statistical significance; $\mathrm{BMD}$, bone mineral density; BV/TV, bone volume/tissue volume ratio; L-dose, low dose (2.5 mg/kg neomangiferin); H-dose, high dose (5 mg/kg neomangiferin).

Histological analysis. Following micro-CT examination, the skulls in each group were placed in an EDTA solution for decalcification. The decalcification solution was replaced every 2 days. The samples were embedded in paraffin and sectioned (section thickness $4 \mu \mathrm{m}$ ). Five consecutive sections were stained with hematoxylin and eosin (H\&E) for each specimen to observe the inflammatory osteolysis necrotic responses. Subsequently, tartrate-resistant acid phosphatase (TRAP) reagent (Sigma, EMD Millipore, Billerica, MA, USA) staining was applied. The sections were placed in the TRAP scanning liquid and incubated for $50 \mathrm{~min}$ at $37^{\circ} \mathrm{C}$, until red wine-colored staining of the osteoclasts was observed under an optical microscope (DM4000B; Leica Microsystems GmbH, Wetzlar, Germany). High-power microscopic examination of images was performed as follows: Each section was subjected to osteoclast counting in five visual fields. Using Image
Pro-Plus 6 software (Media Cybernetics, Inc., Bethesda, MD, USA), TRAP staining analysis (+) was performed to determine the number of osteoclasts and region of osteolysis.

Statistical analysis. Experimental results are represented as the mean \pm standard deviation. Statistical analyses were performed with the SPSS Statistics Package 19.0 (IBM SPSS, Armonk, NY, USA). One-way analysis of variance (ANOVA) was performed to compare groups. If $\mathrm{P}<0.05$ in AVONA, the SNK-q test was used for any pairwise comparisons; $\mathrm{P}<0.05$ was considered to indicate a statistically significant difference.

\section{Results}

Micro-CT scanning. The Micro-CT examination revealed, as shown in Fig. 1A, that the skull surface in the vehicle group 


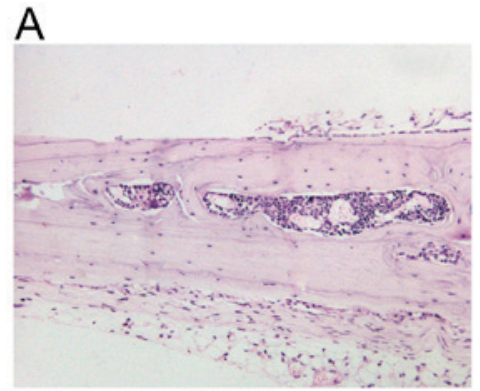

Sham

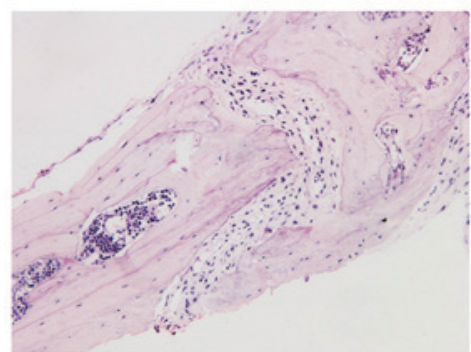

L-dose

B

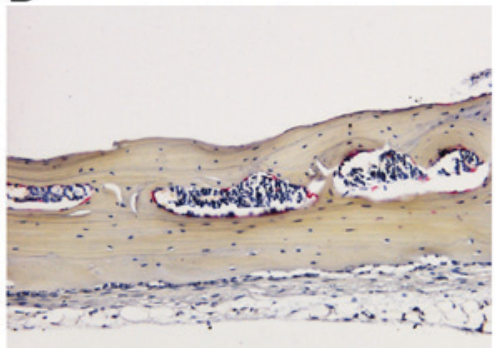

Sham

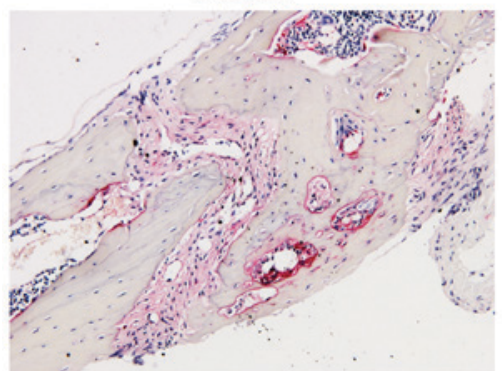

L-dose

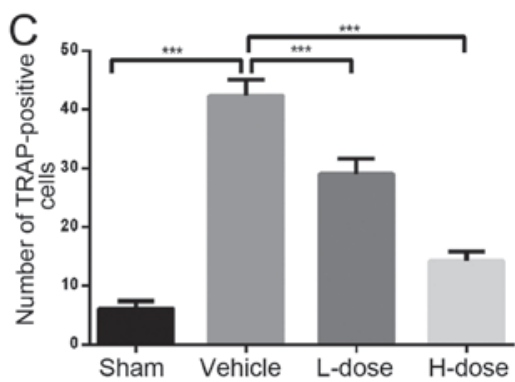

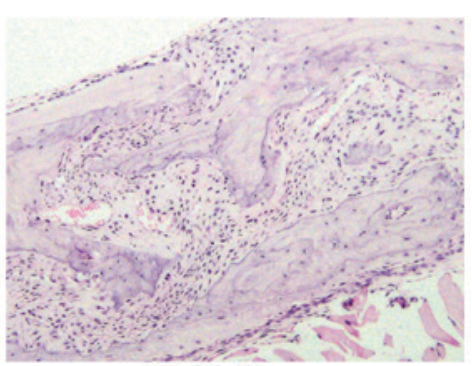

Vehicle

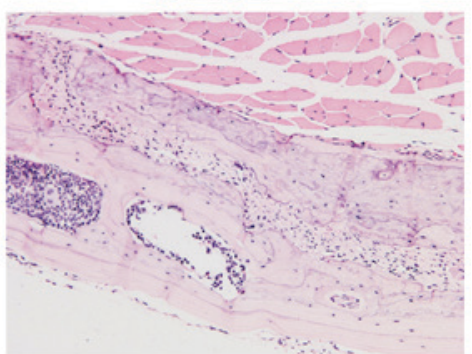

H-dose

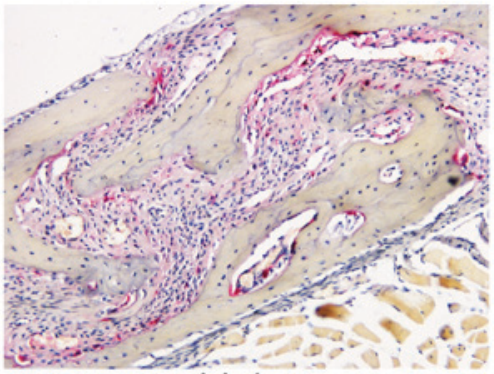

Vehicle

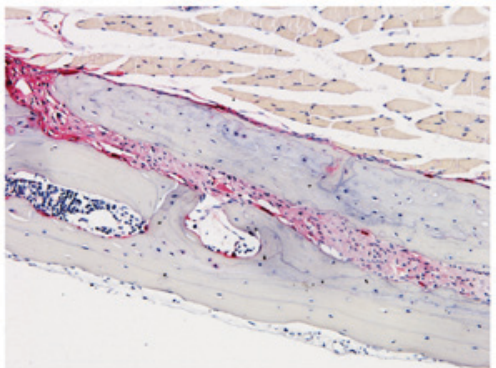

H-dose

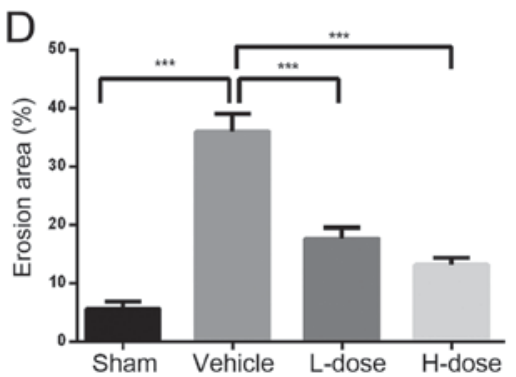

Figure 2. Histological staining of skull sections for each group. (A) Images of hematoxylin and eosin staining (magnification, $\mathrm{x} 100$ ). (B) Images of TRAP staining (magnification, x100). (C) Number of TRAP-positive cells per wide field of view. (D) Erosion area of the calvarial bone within a fixed size range (n=6). One-way analysis of variance and the SNK-q test were performed to determine statistical significance. Results are expressed as the mean \pm standard deviation ( ${ }^{* * *} \mathrm{P}<0.001$, compared with the positive group). TRAP, tartrate-resistant acid phosphatase; L-dose, low dose (2.5 mg/kg neomangiferin); H-dose, high dose $(5 \mathrm{mg} / \mathrm{kg}$ neomangiferin).

underwent severe destruction. By contrast, in the sham group of mice, the skull surface was smooth, without significant osteolysis. In the vehicle group and the low- and high-dose neomangiferin groups, osteolysis occurred to varying degrees. 

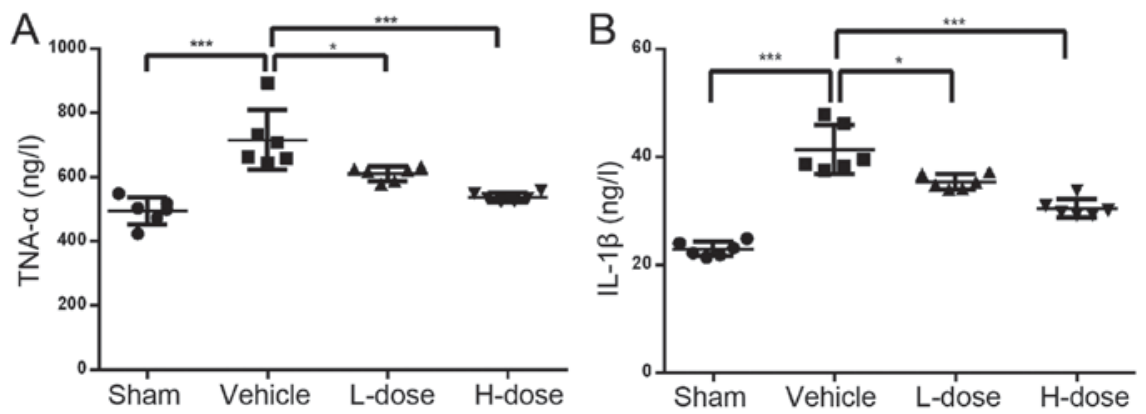

Figure 3. Neomangiferin markedly inhibits the expression of TNF- $\alpha$ and IL-1 $\beta$. Expression levels of (A) TNF- $\alpha$ and (B) IL-1 $\beta$. Comparison was made against the positive control group $\left({ }^{*} \mathrm{P}<0.05,{ }^{* * *} \mathrm{P}<0.001\right)$ TNF- $\alpha$, tumor necrosis factor- $\alpha$; IL-1 $\beta$, interleukin- $1 \beta ;$ L-dose, low dose (2.5 mg $/ \mathrm{kg}$ neomangiferin); H-dose, high dose (5 mg/kg neomangiferin).

Specifically, the surface area of the skull samples was characterized in terms of the depth of bone resorption fossa. The above data showed that the UHMWPE particles induced osteolysis. Neomangiferin significantly inhibited bone destruction, increased BMD (Fig. 1B) and BV/TV (Fig. 1C), and decreased the number of bone resorption pits and total porosity within the skull region of interest (Fig. 1D and E). This analysis confirmed that neomangiferin effectively inhibited the calvarial osteolysis induced by UHMWPE particles. Following neomangiferin treatment, skull destruction was mild, with less damage observed in the high-dose neomangiferin group. The analysis of bone status (Fig. 1B-E) confirmed that the effect of high-dose neomangiferin on bone resorption was significantly greater than that of low-dose neomangiferin.

Histopathological analysis via $H \& E$ staining and TRAP staining. Inflammatory cells, macrophages, and multinucleated osteoclasts were detected in the skulls of mice that received UHMWPE particles (Fig. 2A). In agreement with the micro-CT examination results, TRAP staining showed that a greater number of positively stained cells were observed on the cranial surface of the vehicle group (Fig. 2B). The quantity of TRAP-positive cells was decreased as the neomangiferin dose increased (Fig. 2C). In addition, the histomorphological observations revealed that the area of bone erosion was markedly decreased following neomangiferin application (Fig. 2D). Therefore, these results indicated that neomangiferin inhibited UHMWPE particle-induced osteolysis.

Expression of RANKL, OSCAR, OPG, CTX-1, TNF- $\alpha$ and $I L-1 \beta$. The ELISA results of celiac arterial blood showed that the expression levels of TNF- $\alpha$ and IL- $1 \beta$ in the drug treatment groups were lower those in the positive control group, and the greater the dose, the lower the concentration (Fig. 3A and B). The expression levels of RANKL, OSCAR and CTX-1 were highest in the positive control group compared with those in the other groups $(\mathrm{P}<0.05$; Fig. $4 \mathrm{~A}-\mathrm{C})$. In addition, the expression of CTX-1, RANKL and OSCAR decreased with the increase in neomangiferin dose. The expression of OPG was the lowest in the positive control group. In the high-dose neomangiferin group, the expression of OPG was significantly increased (Fig. 4D). Neomangiferin reduced the expression of RANKL, OSCAR and CTX-1, increased the expression of OPG, and inhibited the expression of proinflammatory factors TNF- $\alpha$ and IL-1 $\beta$. These results were consistent with the previous ELISA analysis, micro-CT examination, and pathological observations. Therefore, these findings suggested that neomangiferin inhibited osteoclast proliferation and differentiation during UHMWPE particle-induced osteolysis, and thereby protected and promoted osteoblast differentiation.

\section{Discussion}

$\mathrm{AL}$ is one of the main reasons for the failure of artificial joint replacements, and prosthesis loosening is considered to be associated with several factors. Studies have shown that the AL of prostheses is associated with extensive infiltration of wear particles. Prosthetic wear particle-induced osteolysis at the bone interface (prosthesis-osteoclastic interface) is a leading cause of loosening of prostheses (21). The prosthesis wear particles can induce an inflammatory reaction involving macrophages in the tissue surrounding the prosthesis, osteoclast activation, and inhibition of osteogenic gene expression (22-24). Inhibition of an early inflammatory response can guarantee implant bone interface stability at the early stage, thereby reducing the influence of wear particles and the physical transfer of the inflammatory media (25). Therefore, inhibiting the inflammatory reaction caused by wear particles and inhibiting osteoclast differentiation are some of the primary means to reduce or prevent the prosthetic loosening. However, there remains a lack of modalities and drugs for the treatment of these problems, and side effects are difficult to manage (26-30). The extract of a Chinese herbal medicine has shown efficacy and mild side effects, thus providing an innovative idea for the treatment of bone destruction-related diseases (31-34). Neomangiferin is a compound from a Chinese medicine substance, which has antioxidative, anti-inflammatory, antiviral, immunoregulatory and antitumor effects $(11,35)$. Neomangiferin is more bioactive than previous forms of mangiferin in order to have a more marked effect on osteolysis (36,37). The traditional Chinese medicine Erxian decoction can promote bone tissue formation, suppress bone resorption and increase bone mineral density, and neomangiferin is one of the main active ingredients. In our previous study, it was found that neomangiferin inhibited osteoclast differentiation in vitro, whereas in vivo neomangiferin inhibited the inflammatory osteolytic effect; however, the possible mechanism remained to be fully elucidated. The mouse model of calvarial inflammatory osteolysis induced by UHMWPE particles used in the present 

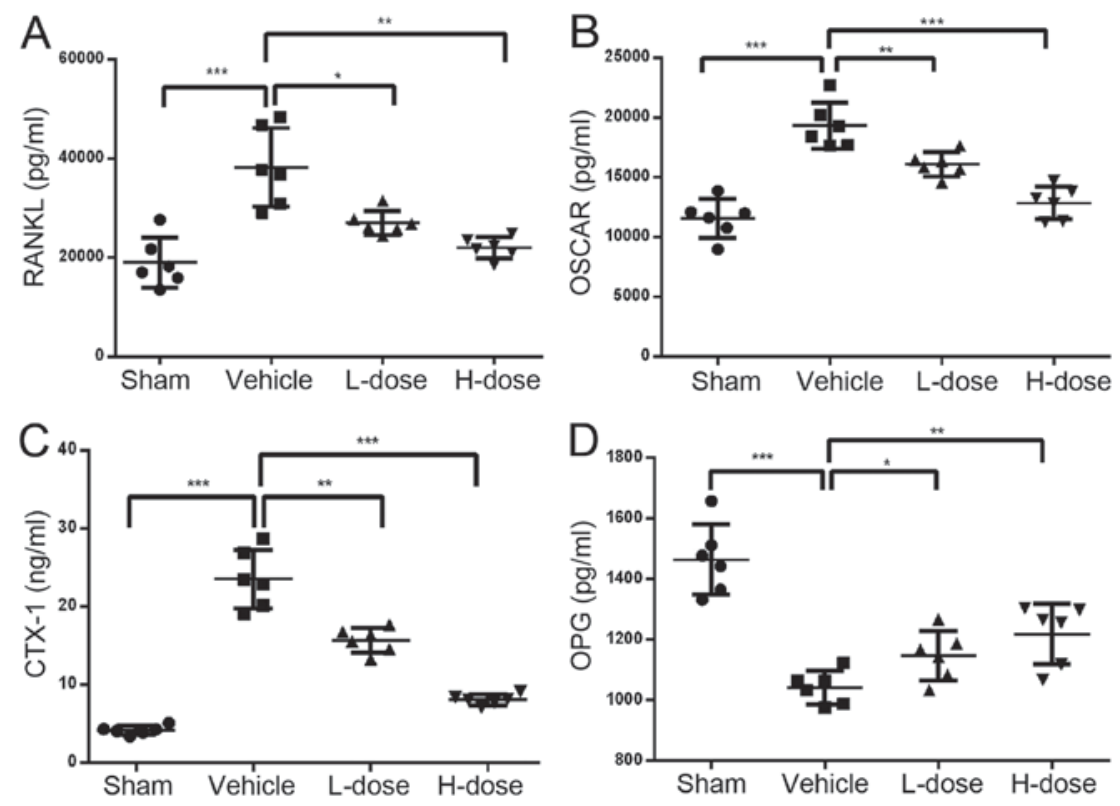

Figure 4. Enzyme-linked immunosorbent assay results. (A) Concentration of RANKL ( $\mathrm{n}=6)$ in the ultra-high-molecular-weight polyethylene particle-induced osteolysis model. (B) Concentration of OSCAR ( $n=6)$. (C) Concentration of CTX-1 ( $n=6)$. (D) Concentration of OPG ( $n=6)$. Results are expressed as the mean \pm standard deviation. $\mathrm{P}<0.05$ indicates statistical significance $\left({ }^{*} \mathrm{P}<0.05,{ }^{* * *} \mathrm{P}<0.01\right.$ and $\left.{ }^{* * * *} \mathrm{P}<0.001\right)$. RANKL, receptor activator of nuclear factor- $\mathrm{kB}$ ligand; OSCAR, osteoclast-related receptor; CTX-1, cross-linked C-telopeptide of type I collagen; OPG, osteoprotegerin; L-dose, low dose ( $2.5 \mathrm{mg} / \mathrm{kg}$ neomangiferin); $\mathrm{H}$-dose, high dose (5 $\mathrm{mg} / \mathrm{kg}$ neomangiferin).

study can simulate joint aseptic osteolysis. Neomangiferin treatment of this tissue environment inhibited osteoclast formation; therefore, it was analyzed for possible curative effects on osteolysis. Specifically, the possible mechanisms underlying the inhibition of UHMWPE particle-induced inflammatory osteolysis were examined.

The results of the micro-CT and histological morphological analyses showed that neomangiferin attenuated UHMWPE particle-induced osteolysis of the skull. In addition, with increased drug concentration, neomangiferin reduced the number of osteoclasts and bone damage. In terms of the mechanism underlying the neomangiferin-driven suppression of osteolysis, the following two assumptions can be made: First, the animal model with neomangiferin injection demonstrated inhibition of osteoclast activity. Prosthesis wear particles stimulate monocyte-derived precursor cell macrophages to differentiate into osteoclasts and enhance their activity, thereby disrupting the dynamic balance of bone resorption and bone formation $(38,39)$. Therefore, the inhibition of osteoclast proliferation and differentiation is a key treatment aim for osteolysis induced by wear particles. In the present study, compared with the negative control group, the number of TRAP-positive cells in the UHMWPE particle group increased significantly. By contrast, the number of TRAP-positive cells in the animal model treated with neomangiferin was significantly lower than in the UHMWPE particle group. In the present study, following neomangiferin treatment, the extent of skull erosion and severity of damage were significantly decreased. Combined with results of the previous experiment, the present data suggested that neomangiferin may inhibit osteoclast formation and differentiation, thus inhibiting osteolysis. Second, neomangiferin may inhibit bone destruction by disrupting the dynamic balance between OPG and RANKL. The combination of RANKL and RANK can regulate the phosphorylation of several downstream signal pathways, including NF-кB, nuclear factor of activated T-cells, and extracellular signal-regulated kinase, in addition to promoting osteoclast proliferation and differentiation, and activating osteoclast maturation to induce bone resorption (40). OPG is expressed in bone marrow stromal cells and osteoblasts. By binding to RANKL, OPG inhibits the binding of RANKL to RANK and prevents the overproduction of osteoclasts (41). Wear particle-induced inflammatory osteolysis can upregulate the expression level of RANKL and inhibit the expression of OPG, thereby inducing osteoclast formation and promoting bone resorption (42-44). Therefore, the dynamic balance of OPG and RANKL affects the wear particle-induced level of osteolysis (45). Compared with the negative control group, the expression levels of OSCAR, RANKL and CTX-1 were increased in the positive control group, whereas that of OPG was decreased. Following 3 weeks of neomangiferin treatment, the expression level of OPG was increased, whereas the expression levels of RANKL, OSCAR and CTX-1 were decreased. Based on the above results, it was hypothesized that neomangiferin suppresses the osteolysis induced by UHMWPE particles by regulating the expression of RANKL and OPG.

Wear particles can induce monocytes to produce cytokines, including IL-1 $\beta$ and TNF- $\alpha$, within the OPG-RANKL-RANK signaling pathway and induce the activation of monocyte-derived macrophage precursor cells $(46,47)$. Additionally, wear particles can transform them into activated osteoclasts, induce fibroblast release of collagenase and prostaglandin E2 associated with bone resorption, and inhibit type I collagen synthesis and osteocalcin in osteoblasts (8), and thus accelerate bone destruction and osteolysis. In the present study, the results of micro-CT, H\&E staining, TRAP staining, and serological ELISA showed that the number of osteoclasts and bone resorption pits in the neomangiferin group decreased with increasing drug concentration. Consequently, the extent of damage 
decreased in the region of mouse calvarial bone resorption. The experiments showed that UHMWPE particles significantly upregulated the expression of inflammatory cytokines, however, neomangiferin significantly inhibited the expression of IL-1 $\beta$ and TNF- $\alpha$ and osteoclastogenesis. TNF- $\alpha$ and IL-1 $\beta$ are the major inflammatory cytokines found in the surrounding tissues of loose prostheses. They are considered as an effective medium for bone resorption. The expression and activity of RANKL are regulated by these proinflammatory cytokines, further supporting their key role in wear particle-induced osteolysis $(41,48,49)$. Furthermore, the inhibitory effect was dose-dependent. Therefore, neomangiferin may inhibit the inflammatory bone resorption induced by UHMWPE particles by reducing the secretion of proinflammatory cytokines and by reducing osteoclastogenesis.

In conclusion, the in vivo experiments showed that neomangiferin inhibited the activation of osteoclasts and thereby influenced the UHMWPE particle-induced osteolystic process. However, the possible mechanism is to be determined in future experiments. In this process, the anti-inflammatory effects of neomangiferin and its ability to modulate the expression of RANKL and OPG may be important. These results show that neomangiferin may be a promising treatment of wear particle-induced inflammatory osteolysis.

\section{Acknowledgements}

Not applicable.

\section{Funding}

This study was supported by the Guangxi Natural Science Foundation (grant no. 2017GXNSFAA198258).

\section{Availability of data and materials}

The datasets used and/or analyzed during the present study are available from the corresponding author on reasonable request.

\section{Authors' contributions}

J-MZ and JY designed the current study; H-TW, JL, S-TM, W-YF, QW and H-YZ performed the experiments, analyzed the data and prepared the figures; H-TW wrote the manuscript. All authors reviewed the article.

\section{Ethics approval and consent to participate}

The animal protocol was approved by the Animal Ethics Committee of Guangxi Medical University (approval no. 201707006), and the Guidelines for Care and Use of Laboratory Animals were strictly followed.

\section{Consent for publication}

Not applicable.

\section{Competing interests}

The authors confirm that they have no competing interests.

\section{References}

1. Sabokbar A, Kudo O and Athanasou NA: Two distinct cellular mechanisms of osteoclast formation and bone resorption in periprosthetic osteolysis. J Orthop Res 21: 73-80, 2003.

2. Shin DK, Kim MH, Lee SH, Kim TH and Kim SY: Inhibitory effects of luteolin on titanium particle-induced osteolysis in a mouse model. Acta Biomater 8: 3524-3531, 2012.

3. Goodman SB, Gibon E, Pajarinen J, Lin TH, Keeney M, Ren PG, Nich C, Yao Z, Egashira K, Yang F and Konttinen YT: Novel biological strategies for treatment of wear particle-induced periprosthetic osteolysis of orthopaedic implants for joint replacement. J R Soc Interface 11: 20130962, 2014.

4. Goodman SB, Gibon E and Yao Z: The basic science of periprosthetic osteolysis. Instr Course Lect 62: 201-206, 2013.

5. Warme BA, Epstein NJ, Trindade MC, Miyanishi K, Ma T, Saket RR, Regula D, Goodman SB and Smith RL: Proinflammatory mediator expression in a novel murine model of titanium-particle-induced intramedullary inflammation. J Biomed Mater Res B Appl Biomater 71: 360-366, 2004.

6. Yang SY, Wu B, Mayton L, Mukherjee P, Robbins PD, Evans CH and Wooley PH: Protective effects of IL-1Ra or vIL-10 gene transfer on a murine model of wear debris-induced osteolysis. Gene Ther 11: 483-491, 2004.

7. Pacifici R: Role of T cells in ovariectomy induced bone loss-revisited. J Bone Miner Res 27: 231-239, 2012.

8. Jonitz-Heincke A, Lochner K, Schulze C, Pohle D, Pustlauk W, Hansmann D and Bader R: Contribution of human osteoblasts and macrophages to bone matrix degradation and proinflammatory cytokine release after exposure to abrasive endoprosthetic wear particles. Mol Med Rep 14: 1491-1500, 2016.

9. Yagil-Kelmer E, Kazmier P, Rahaman MN, Bal BS, Tessman RK and Estes DM: Comparison of the response of primary human blood monocytes and the U937 human monocytic cell line to two different sizes of alumina ceramic particles. J Orthop Res 22: 832-838, 2004

10. Petit A, Mwale F, Antoniou J, Zukor DJ and Huk OL: Effect of bisphosphonates on the stimulation of macrophages by alumina ceramic particles: A comparison with ultra-high-molecular-weight polyethylene. J Mater Sci Mater Med 17: 667-673, 2006.

11. Lim SM, Kang GD, Jeong JJ, Choi HS and Kim DH: Neomangiferin modulates the Th17/Treg balance and ameliorates colitis in mice. Phytomedicine 23: 131-140, 2016.

12. Li J, Chen L, Wu H, Lu Y, Hu Z, Lu B, Zhang L, Chai Y and Zhang J: The mixture of salvianolic acids from salvia miltiorrhiza and total flavonoids from anemarrhena asphodeloides attenuate sulfur mustard-induced injury. Int J Mol Sci 16: 24555-24573, 2015.

13. Seo CS, Ha H, Kim YJ and Jungb JY: HPLC-pDA simultaneous determination and protective effect of Anemarrhena asphodeloides against acute renal failure. Nat Prod Commun 9: 829-832, 2014.

14. Zhou C, Zhou J, Han N, Liu Z, Xiao B and Yin J: Beneficial effects of neomangiferin on high fat diet-induced nonalcoholic fatty liver disease in rats. Int Immunopharmacol 25: 218-228, 2015.

15. Zhu X, Gao JJ, Landao-Bassonga E, Pavlos NJ, Qin A, Steer JH, Zheng MH, Dong Y and Cheng TS: Thonzonium bromide inhibits RANKL-induced osteoclast formation and bone resorption in vitro and prevents LPS-induced bone loss in vivo. Biochem Pharmacol 104: 118-130, 2016.

16. Zhu X, Gao J, Ng PY, Qin A, Steer JH, Pavlos NJ, Zheng MH, Dong Y and Cheng TS: Alexidine dihydrochloride attenuates osteoclast formation and bone resorption and protects against LPS-induced osteolysis. J Bone Miner Res 31: 560-572, 2016.

17. Chen H, Guo T, Wang D and Qin R: Vaccaria hypaphorine impairs RANKL-induced osteoclastogenesis by inhibition of ERK, p38, JNK and NF- $\kappa \mathrm{B}$ pathway and prevents inflammatory bone loss in mice. Biomed Pharmacother 97: 1155-1163, 2018.

18. Song F, Wei C, Zhou L, Qin A, Yang M, Tickner J, Huang Y, Zhao J and $\mathrm{Xu}$ J: Luteoloside prevents lipopolysaccharide-induced osteolysis and suppresses RANKL-induced osteoclastogenesis through attenuating RANKL signaling cascades. J Cell Physiol 233: 1723-1735, 2018.

19. Al-Quhali AM, Sun Y, Bai X, Jin Z and Yu G: Surgical modification of the murine calvaria osteolysis model. Biomed Res Int 2015: 802697, 2015. 
20. Wedemeyer C, Xu J, Neuerburg C, Landgraeber S, Malyar NM, von Knoch F, Gosheger G, von Knoch M, Löer F and Saxler G: Particle-induced osteolysis in three-dimensional micro-computed tomography. Calcif Tissue Int 81: 394-402, 2007.

21. Sun Q, Fu Y, Sun A, Shou Y,Zheng M,Li X and Fan D: Correlation of E-selectin gene polymorphisms with risk of ischemic stroke A meta-analysis. Neural Regenerat Res 6: 1731-1735, 2011.

22. Chiu R, Ma T, Smith RL and Goodman SB: Ultrahigh molecular weight polyethylene wear debris inhibits osteoprogenitor proliferation and differentiation in vitro. J Biomed Mater Res A 89: 242-247, 2009.

23. Chiu R, Ma T, Smith RL and Goodman SB: Polymethylmethacry late particles inhibit osteoblastic differentiation of bone marrow osteoprogenitor cells. J Biomed Mater Res A 77: 850-856, 2006.

24. Kadoya Y, Revell PA, al-Saffar N, Kobayashi A, Scott G and Freeman MA: Bone formation and bone resorption in failed total joint arthroplasties: Histomorphometric analysis with histochemical and immunohistochemical technique. J Orthop Res 14 473-482, 1996

25. Schmalzried TP, Kwong LM, Jasty M, Sedlacek RC, Haire TC, O'Connor DO, Bragdon CR, Kabo JM, Malcolm AJ and Harris WH: The mechanism of loosening of cemented acetabular components in total hip arthroplasty. Analysis of specimens retrieved at autopsy. Clin Orthop Relat Res: 274, 60-78, 1992

26. Kotian P, Boloor A and Sreenivasan S: Study of adverse effect profile of parenteral zoledronic acid in female patients with osteoporosis. J Clin Diagn Res 10: OC04-OC06, 2016.

27. Beaudoin C, Jean S, Bessette L, Ste-Marie LG, Moore L and Brown JP: Denosumab compared to other treatments to prevent or treat osteoporosis in individuals at risk of fracture: A systematic review and meta-analysis. Osteoporos Int 27: 2835-2844, 2016.

28. Tsubaki M, Komai M, Itoh T, Imano M, Sakamoto K, Shimaoka H, Takeda T, Ogawa N, Mashimo K, Fujiwara D, et al: Nitrogen-containing bisphosphonates inhibit RANKL- and $\mathrm{M}-\mathrm{CSF}$-induced osteoclast formation through the inhibition of ERK1/2 and Akt activation. J Biomed Sci 21: 10, 2014.

29. He LG, Li XL, Zeng XZ, Duan H, Wang S, Lei LS, Li XJ and Liu SW: Sinomenine induces apoptosis in RAW 264.7 cell-derived osteoclasts in vitro via caspase-3 activation. Acta Pharmacol Sin 35: 203-210, 2014.

30. Zheng M, Ge Y, Li H, Yan M, Zhou J and Zhang Y: Bergapten prevents lipopolysaccharide mediated osteoclast formation, bone resorption and osteoclast survival. Int Orthop 38: 627-634, 2014.

31. Newman DJ and Cragg GM: Natural products as sources of new drugs from 1981 to 2014. J Nat Prod 79: 629-661, 2016.

32. Wang J, Fu B, Lu F, Hu X, Tang J and Huang L: Inhibitory activity of linarin on osteoclastogenesis through receptor activator of nuclear factor $\kappa \mathrm{B}$ ligand-induced $\mathrm{NF}-\kappa \mathrm{B}$ pathway. Biochem Biophys Res Commun 495: 2133-2138, 2018.

33. Liu G, Ma C, Wang P, Zhang P, Qu X, Liu S, Zhai Z, Yu D, Gao J, Liang J, et al: Pilose antler peptide potentiates osteoblast differentiation and inhibits osteoclastogenesis via manipulating the NF- $\kappa \mathrm{B}$ pathway. Biochem Biophys Res Commun 491: 388-395, 2017.

34. Nie S, Xu J, Zhang C, Xu C, Liu M and Yu D: Salicortin inhibits osteoclast differentiation and bone resorption by down-regulating JNK and NF- $\mathrm{B} / \mathrm{NFATc1}$ signaling pathways. Biochem Biophys Res Commun 470: 61-67, 2016.

35. HencI,KokotkiewiczA,ŁuczkiewiczP,BrylE,ŁuczkiewiczMand Witkowski JM: Naturally occurring xanthone and benzophenone derivatives exert significant anti-proliferative and proapoptotic effects in vitro on synovial fibroblasts and macrophages from rheumatoid arthritis patients. Int Immunopharmacol 49: 148-154, 2017.
36. Liao HL, Wu QY, HU HG, Zang ZH, Song L and Yang Q: Structure modification of mangiferin. West China J Pharma Sci: 23, 385-387, 2008

37. Hong YF, Han GY and Guo XM: Isolation and structure determination of xanthone glycosides of Anemarrhena asphodeloides. Yao Xue Xue Bao 32: 473-475, 1997 (In Chinese).

38. Broadhead ML, Clark JC, Dass CR, Choong PF and Myers DE: Therapeutic targeting of osteoclast function and pathways. Expert Opin Ther Targets 15: 169-181, 2011.

39. Landgraeber S, Quint U, Classen T and Totsch M: Senescence in cells in aseptic loosening after total hip replacement. Acta Biomater 7: 1364-1368, 2011.

40. Boyle WJ, Simonet WS and Lacey DL: Osteoclast differentiation and activation. Nature 423: 337-342, 2003

41. Zhai Z, Qu X, Li H, Yang K, Wan P, Tan L, Ouyang Z, Liu X, Tian B, Xiao F, et al: The effect of metallic magnesium degradation products on osteoclast-induced osteolysis and attenuation of NF- $\kappa$ B and NFATc1 signaling. Biomaterials 35: 6299-6310, 2014.

42. Cadosch D, Gautschi OP, Chan E, Simmen HP and Filgueira L: Titanium induced production of chemokines CCL17/TARC and CCL22/MDC in human osteoclasts and osteoblasts. J Biomed Mater Res A 92: 475-483, 2010.

43. Jämsen E, Kouri VP, Olkkonen J, Cör A, Goodman SB, Konttinen YT and Pajarinen J: Characterization of macrophage polarizing cytokines in the aseptic loosening of total hip replacements. J Orthop Res 32: 1241-1246, 2014.

44. Jablonski H, Rekasi H and Jäger M: The influence of calcitonin gene-related peptide on markers of bone metabolism in MG-63 osteoblast-like cells co-cultured with THP-1 macrophage-like cells under virtually osteolytic conditions. BMC Musculoskelet Disord 17: 199, 2016.

45. Park SJ, Lee EJ, Kim YH, Shin JE and Kang YH: Inhibitory effects of gossypin on RANKL-induced osteoclast differentiation and bone resorption in murine macrophages (LB364). FASEB J 28, 2014.

46. Nich C, Takakubo Y, Pajarinen J, Ainola M, Salem A, Sillat T, Rao AJ, Raska M, Tamaki Y, Takagi M, et al: Macrophages-Key cells in the response to wear debris from joint replacements. J Biomed Mater Res A 101: 3033-3045, 2013

47. Ingham $\mathrm{E}$ and Fisher J: The role of macrophages in osteolysis of total joint replacement. Biomaterials 26: 1271-1286, 2005.

48. Lin TH, Yao Z, Sato T, Keeney M, Li C, Pajarinen J, Yang F, Egashira K and Goodman SB: Suppression of wear-particle-induced pro-inflammatory cy tokine and chemokine production in macrophages via NF- $\mathrm{BB}$ decoy oligodeoxynucleotide: A preliminary report. Acta Biomater 10: 3747-3755, 2014.

49. Liu X, Zhu S, Cui J, Shao H, Zhang W, Yang H, Xu Y, Geng D and $\mathrm{Yu}$ L: Strontium ranelate inhibits titanium-particle-induced osteolysis by restraining inflammatory osteoclastogenesis in vivo. Acta Biomater 10: 4912-4918, 2014.

c) (i) $($ This work is licensed under a Creative Commons Attribution-NonCommercial-NoDerivatives 4.0 International (CC BY-NC-ND 4.0) License. 